\title{
The classification of movement intention through machine learning models: The identification of significant time-domain EMG features
}

\author{
Ismail Mohd Khairuddin ${ }^{1,2}$, Shahrul Na'im Sidek ${ }^{\text {Corresp., }}{ }^{2}$, Anwar P.P. Abdul Majeed ${ }^{1}$, Mohd Azraai Mohd Razman ${ }^{1}$, \\ Asmarani Ahmad Puzi ${ }^{2}$, Hazlina Md Yusof ${ }^{2}$ \\ ${ }^{1}$ Faculty of Manufacturing \& Mechatronics Engineering Technology, Innovative Manufacturing, Mechatronics and Sports Laboratory, Universiti Malaysia \\ Pahang, Pekan, Pahang, Malaysia \\ 2 Department of Mechatronics, Kulliyyah of Engineering, Biomechatronics Research Laboratory, International Islamic University, Gombak, Selangor, \\ Malaysia \\ Corresponding Author: Shahrul Na'im Sidek \\ Email address: snaim@iium.edu.my
}

Electromyography (EMG) signal is one of the extensively utilised biological signals for predicting human motor intention, which is an essential element in human-robot collaboration platforms. Studies on motion intention prediction from EMG signals have often been concentrated on either classification and regression models of muscle activity. In this study, we leverage the information from the EMG signals, to detect the subject's intentions in generating motion commands for a robot-assisted upper limb rehabilitation platform. The EMG signals are recorded from ten healthy subjects' biceps muscle, and the movements of the upper limb evaluated are voluntary elbow flexion and extension along the sagittal plane. The signals are filtered through a fifth-order Butterworth filter. A number of features were extracted from the filtered signals namely waveform length (WL), mean absolute value (MAV), root mean square (RMS), standard deviation (SD), minimum (MIN) and maximum (MAX). Several different classifiers viz. Linear Discriminant Analysis (LDA), Logistic Regression (LR), Decision Tree (DT), Support Vector Machine (SVM) and kNearest Neighbour (k-NN) were investigated on its efficacy to accurately classify the preintention and intention classes based on the significant features identified (MIN and MAX) via Extremely Randomised Tree feature selection technique. It was observed from the present investigation that the DT classifier yielded an excellent classification with a classification accuracy of $100 \%, 99 \%$ and $99 \%$ on training, testing and validation dataset, respectively based on the identified features. The findings of the present investigation are non-trivial towards facilitating the rehabilitation phase of patients based on their actual capability and hence, would eventually yield a more active participation from them. 
1 The Classification of Movement Intention through

2 Machine Learning Models:

3 The Identification of Significant EMG Time-Domain

4 features

5

6

7

8

9

10

11

12

13

14

15

16

17

18

19

20

21

22

23

24

25

26

27

28

29

30

31

32

33

34

35

36

37

38

Ismail Mohd Khairuddin ${ }^{1,2}$, Shahrul Na'im Sidek ${ }^{1}$, Anwar P.P. Abdul Majeed ${ }^{2}$, Mohd Azraai

Mohd Razman ${ }^{2}$, Asmarani Ahmad Puzi ${ }^{1}$, and Hazlina Md Yusof ${ }^{1}$

${ }^{1}$ Department of Mechatronics, Kulliyyah of Engineering, International Islamic University

Malaysia, Biomechatronics Research Laboratory, Gombak, Selangor, Malaysia

${ }^{2}$ Faculty of Manufacturing \& Mechatronics Engineering Technology, Universiti Malaysia

Pahang, Innovative Manufacturing, Mechatronics and Sports Laboratory, Pekan, Pahang,

Malaysia

Corresponding Author:

Shahrul Na'im Sidek ${ }^{1}$

Gombak, Selangor, 53100, Malaysia

Email address: snaim@iiium.edu.my

\section{Abstract}

Electromyography (EMG) signal is one of the extensively utilised biological signals for predicting human motor intention, which is an essential element in human-robot collaboration platforms. Studies on motion intention prediction from EMG signals have often been concentrated on either classification and regression models of muscle activity. In this study, we leverage the information from the EMG signals, to detect the subject's intentions in generating motion commands for a robot-assisted upper limb rehabilitation platform. The EMG signals are recorded from ten healthy subjects' biceps muscle, and the movements of the upper limb evaluated are voluntary elbow flexion and extension along the sagittal plane. The signals are filtered through a fifth-order Butterworth filter. A number of features were extracted from the filtered signals namely waveform length (WL), mean absolute value (MAV), root mean square (RMS), standard deviation (SD), minimum (MIN) and maximum (MAX). Several different classifiers viz. Linear Discriminant Analysis (LDA), Logistic Regression (LR), Decision Tree (DT), Support Vector Machine (SVM) and k-Nearest Neighbour (k-NN) were investigated on its efficacy to accurately classify the pre-intention and intention classes based on the significant features identified (MIN and MAX) via Extremely Randomised Tree feature selection technique. It was observed from the present investigation that the DT classifier yielded an excellent classification with a classification accuracy of $100 \%, 99 \%$ and $99 \%$ on training, testing and 
validation dataset, respectively based on the identified features. The findings of the present investigation are non-trivial towards facilitating the rehabilitation phase of patients based on their actual capability and hence, would eventually yield a more active participation from them.

\section{Introduction}

Robot-assisted platforms have been introduced over the last two decades in order to complement the work of physiotherapists and have been shown to provide positive results in assisting subjects to regain their activities of daily living (ADL) (Brackenridge et al., 2016; Chen et al., 2016; Fasoli, 2016). Amongst the advantages of the utilisation of assist-based robotics therapy, it in its ability to provide autonomous training where subjects are involved in the repeated exercise of goal-directed tasks that in turn, leads to improvements in their motor function (Peternel et al., 2016; Taha et al., 2019). The importance of implementing such robot-assist platform could reduce the burden of the physiotherapist as well as facilitating active rehabilitation process of the subjects (Rahman, Ochoa-Luna \& Saad, 2015a; Huang, Tu \& He, 2016).

Even though the use of such robot-assist platforms could significantly facilitate the rehabilitation process, nonetheless, the key to rehabilitation recovery is often dependent on the subjects' natural capability (Rahman et al., 2015; Zimmermann et al., 2015). In order to provide assistance and keep motivating the subjects in performing the prescribed tasks programmed on the robotic platform, the selection of a suitable controller is essential. Several controllers have thus far been applied to the robot-platform to aid the patient in the movement recovery process (Rahman, Ochoa-Luna \& Saad, 2015a; Zimmermann et al., 2015; Proietti et al., 2016). It should be noted that in order to improve the interaction between the subject and the robotic-assisted platform, the capturing or detection of physiological motion intention data is non-trivial, as a substantial effort is required by the patient to complete the prescribed exercise. Through the identification of the motion intention, a suitable amount of assistive force could be regulated by the controller of the robotic platform to facilitate the completion of the prescribed task.

To date, different methods have been developed to detect or capture the intent of subjects. For instance, capturing intention-based triggering is one of the simplest and most widely used (Marchal-Crespo \& Reinkensmeyer, 2009; Proietti et al., 2016; Bi, Feleke \& Guan, 2019). This triggering signal can be either force, velocity, time thresholds, or even motion intent based on electromyography (EMG) or electroencephalography (EEG) (Krebs et al., 2003; Colombo et al., 2005; Loureiro et al., 2009; Rechy-Ramirez \& Hu, 2015; Bi, Feleke \& Guan, 2019).

Nevertheless, it is worth noting that triggering signals are rather artificial and does not reflect the actual intention of the subject. Conversely, the processing of EEG signals is rather complex. An effective and attractive strategy in acquiring the movement intention is surface electromyogram (EMG) signals (Bi, Feleke \& Guan, 2019)(Guidali et al., 2013). This method could allow the robotic system to be activated prior to the actual motion of the subject. It has been reported that EMG can provide the information of muscle activation between $40 \mathrm{~ms}$ to $100 \mathrm{~ms}$ (Winter, 1990; Tommaso Lenzi, Stefano Marco Maria De Rossi, Nicola Vitiello Maria, 2012; Kosaki et al., 2017). 
79 In order to use the EMG signals as an input for detecting the motion intention, these signals need

80 to be extracted first. For instance, AlOmari and Liu (2014) applied several methods to extract the 81 features such as sample entropy (SampEnt), root mean square (RMS), myopulse percentage rate

82 (MYOP), and difference absolute standard deviation value (DASDV) in order to classify the 83 forearm muscle activity. Other researchers have exploited different time-domain features, 84 namely waveform length (WL), mean absolute value (MAV), zero crossing (ZC), and slope-sign 85 change (SSC) (Kamavuako, Scheme \& Englehart, 2014) to distinguish the movement of the 86 forearm.

87 In terms of the classification of the aforesaid signals, several methods have been employed. 88 Bhattacharya, Sarkar and Basak (2017) investigated the efficacy of Subspace Discriminant 89 (SDE) Ensemble apart from $k$-Nearest Neighbour $(k-\mathrm{NN})$, Linear Discriminant Analysis (LDA) 90 and Quadratic Discriminant Analysis (QDA) in classifying different set of EMG features for hand movement recognition. It was shown from the study that the SDE classifier could yield the highest classification accuracy of $83.33 \%$ for training dataset. Oskoei and $\mathrm{Hu}$ (2008) evaluated the performance of different SVM models (kernels) in classifying upper limb motions using EMG signals against LDA and artificial neural networks (ANN). The kernels evaluated in the study were radial-basis, linear, polynomial and sigmoid. It was shown from the study that the average accuracy for all kernels was approximately $95.57 \%$ followed by LDA and ANN. To the best of the authors' knowledge, although there are studies that have employed different statistical EMG time-domain features, nonetheless limited studies have highlighted the significance of the features extracted. Therefore, this paper aims at identifying significant EMG time-domain features that could facilitate in the detection of motion intention from the subject that in turn will provide a seamless assistance for robotics-based rehabilitation. The remainder of this paper is organised as follows: Section 2 describes the approach taken for detecting intention via the EMG signals. Section 3 describes the classification methods employed on the EMG signals. Section 4 presents the result, and Section 5 draws the conclusion of the present work as well as provide the future direction of the present investigation.

\section{Materials \& Methods}

\section{Participants}

Ten healthy subjects (four females and six males) with no neurological or orthopaedic impairment from the Department of Mechatronics Engineering of International Islamic University Malaysia (IIUM) participated in the present investigation. The participants were between the age range of 22 and 26, with a mean and standard deviation of 24.4 years, and 3.27 years, respectively. In addition, the mean weight and height of the subjects are $68 \mathrm{~kg}$ and 167 $\mathrm{cm}$, respectively. The experiment was conducted at a dedicated room at Biomechatronic Research Laboratory of IIUM. The experiments were conducted with the approval of the International Islamic University Malaysia Research Ethics Community (IREC) 659, and all participants gave verbal and written informed consent prior to the experiment. 
119

120

121

122

123

124

125

126

127

128

129

130

131

132

133

134

135

136

137

138

139

140

141

142

143

144

145

146

147

148

149

150

151

152

153

154

155

156

157

158

\section{Intention Recognition System}

Fig 1. illustrates the proposed framework for detecting the motion intention of the subject. The intention recognition system utilised in this present study consists of three main components, the PC workstation (blue-dashed box), where the main program of the overall system is linked with the second component, i.e., the DAQ system (red-dashed box). The last component is the collection of sensors namely torque sensor, potentiometer as well as the EMG electrode (purpledashed box). The complete robotic platform which consists of the aforementioned components that are used in the present study is depicted in Fig 2.

\section{Figure 1. Block Diagram for data acquisition}

Figure 2. Robotic Platform

\section{Data Acquisition and Processing}

The electromyography signal is captured via the g.USBamp signal amplifier (g.tec Medical Engineering $\mathrm{GmbH}$ ). This signal is then sent to the PC workstation for processing purpose. In order to record the EMG signal, each subject was instructed to perform three sets of flexion movements (from $0^{\circ}$ to $45^{\circ}$ with an interval of $2 \mathrm{~s}$ before further moving from $45^{\circ}$ to $90^{\circ}$ ). These movements transpired in the sagittal plane with the upper arm being held horizontally $\left(0^{\circ}\right)$ and the subject was asked to maintain as still in the upright sitting position. During the experimental testing, each subject needs to intentionally move the robotic arm platform of the robot rehabilitation system. The electromyographic signals were sampled at $1.2 \mathrm{kHz}$. The biosignals were recorded using two disposable surface electrodes placed in a configuration suggested by the manufacturer of the signal amplifier (one on the biceps, and one on the bone which is acting as a ground) as depicted in Fig. 3. A bandpass filter was applied to filter the EMG signals between the range of $5 \mathrm{~Hz}$ to $500 \mathrm{~Hz}$. The angular motion (angle) of the prescribed movements is captured via the potentiometer that is processed via Humsoftt MF624 at a sampling rate of $0.001 \mathrm{~Hz}$.

Figure 3. Electrode placement

\section{Feature Extraction}

The EMG signals were segmented into two distinct sections, namely pre-intention, and intention as depicted in Fig 4. The intention signal is recorded based on the definition of muscle burst which transpires between $40 \mathrm{~ms}$ to $100 \mathrm{~ms}$ prior to any muscle activities (Winter, 1990; Kosaki et al., 2017). The segmentation groups are based on the aforesaid information. The features of each signal of this segmentation are extracted by using time-domain (TD) technique as it is obtained from the signals' amplitude (Veer \& Sharma, 2016; Spiewak, 2018) In each, $x_{i}$ is $i_{t h}$ sample of EMG signal amplitude and $L$ is the length of the analysis window for computing the features. The TD 
159 techniques applied in this research is described as follows (Purushothaman \& Ray, 2014; Naik, 160 2015; Rahman, Ochoa-Luna \& Saad, 2015a)

161

Figure 4. A sample of the EMG and angular position for a single movement.

163

164

165

166

167

168

169

170

171

172

173

174

175

176

177

178

179

180

181

182

183

184

185

186

187

Waveform Length: Is the cumulative length of the waveform over the segment. It specifies a measure of waveform amplitude, frequency, and duration all within a single parameter

$$
\mathrm{WL}=\sum_{i=1}^{L-1}\left|x_{i+1}-x_{i}\right|
$$

Mean Absolute Value: It signifies the area under the EMG signal once it has been rectified. The MAV is used as a measure of the amplitude of the EMG signal.

$$
\mathrm{MAV}=\frac{1}{L} \sum_{i=1}^{L}\left|x_{i}\right|
$$

Root Mean Square: The RMS denotes the square root of the average power of the EMG signal for a given period of time.

$$
\mathrm{RMS}=\sqrt{\frac{1}{L} \sum_{i=1}^{L} x_{i}^{2}}
$$

Standard Deviation: The SD of a set of data is the square root of the variance, where $\mu$ refers to the mean of the sample.

$$
\mathrm{SD}=\sqrt{\frac{1}{L-1} \sum_{i=1}^{L}\left|x_{i}-\mu\right|^{2}}
$$

$$
\mu=\frac{1}{L} \sum_{i=1}^{L} x_{i}
$$

Maximum amplitude: Maximum value of the EMG signal

$$
\operatorname{Max}=\max \left|x_{i}\right|
$$

\section{Minimum amplitude: Minimum value of the EMG signal}

$$
\operatorname{Min}=\min \left|x_{i}\right|
$$

The rationale for utilizing the time-domain features is primarily due to its swift computation as the features do not require any transformation on the raw EMG data (Spiewak, 2018). Moreover, it is worth noting that the time-domain features have been widely used in both medical and engineering types of research and practices (Rahman, Ochoa-Luna \& Saad, 2015b).

\section{Feature Selection}

The selection of features is used to reduce the number of features that do not significantly contribute to the classification of the intention of the subject. In the present study, the best 
188 feature for the classification process is attained by means of an Extremely Randomised Tree 189 (ERT) technique. The ERT is a tree-based ensemble learning technique that combines the results 190 of multiple de-correlated decision trees collected (Geurts, Ernst \& Wehenkel, 2006). The 191 entropy-based information gain is essentially used as the decision criteria for the significant 192 features. Fig. 5 illustrate the bar graph for each of the extracted features. It is evident from Fig. 5; 193 the most significant features identified are the MIN and MAX, respectively.

194

195

196

197

198

199

200

201

202

203

204

205

206

207

208

209

210

211

212

213

214

215

216

217

218

219

220

221

Figure 5. Bar graph of feature importance

\section{Classification}

Classification is the last yet essential step employed in order to achieve the objectives of the present study. In this study, five different models of classification approaches are utilised on the time-series or time-domain (TD) features extracted, namely Linear Discriminant Analysis (LDA), Logistic Regression (LR), Decision Tree (DT), $k$-Nearest Neighbour $(k$-NN), and Support Vector Machine (SVM). A total of 45358 (15\%) of the dataset was used for testing, $211668(70 \%)$ for training and 45357 (15\%) was used for independent testing (validation). The five-fold cross-validation technique was used on the train the models, i.e., on the training dataset as this method has been reported to mitigate the issue of over-fitting (Razman et al., 2019). The development of the models was carried out via Scikit-learn Python Library on Spyder 4.1.4 IDE. It is worth noting that the models are developed based on its default hyperparameters as per the utilised library.

\section{Performance Matrix}

The classifier's performance is evaluated based on its classification accuracy (CA), precision, recall, F1-score, as well as the confusion matrix. Fig. 6 below demonstrates the confusion matrix as an evaluation of the predictive models.

Figure 6. Confusion matrix

$$
\begin{aligned}
& \text { Accuracy }=\left(\frac{T P+T N}{T P+T N+F P+F N}\right) \times 100 \\
& \text { Precision }=\left(\frac{T P}{T P+F P}\right) \times 100 \\
& \text { Recall }=\left(\frac{T P}{T P+F N}\right) \times 100
\end{aligned}
$$




$$
F_{1}=2\left(\frac{\text { Precision } \bullet \text { Recall }}{\text { Precision }+ \text { Recall }}\right) \times 100
$$

223 From the equations above, the accuracy is defined as the overall classification rate and on how

224

225

226

227

228

229

230

231

232

233

234

235

236

237

238

239

240

241

242

243

244

245

246

247

248

249

250

251

252

253

254

255

256

257

258 well the classifier predicts the classes. The precision evaluates the prediction rate of correctly predicted classes, conversely recall is the ratio of the positive classes that are correctly categorised. The F1 is the harmonic mean that is generated between precision and recall by multiplying the scale by 2 . The F1 score provides the quality of the prediction especially for uneven class distribution as exhibited in the present study.

\section{Results}

Fig 7 illustrates the performance of the evaluated classifier, in terms of classification accuracy (CA) based on all features, whilst Fig 8 illustrates the CA based on selected significant features identified via the ERT algorithm. The details on the other performance measures are tabulated in Table 1 and Table 2, respectively.

Figure 7. Classification accuracy for each model for all features Table 1 Classifier models performance with all features

Figure 8. Classification accuracy for each model for the selected features

Table 2 Classifier models performance with the selected features

\section{Discussion}

Tables 1, and 2 recorded the classification performance for the different classifiers evaluated with respect to all features and selected. As tabulated in table 1, it could be seen that the DT classifier model provides the highest accuracy during training with a CA of $100 \%$ in comparison to other classifier models evaluated. Nonetheless, in the event that a new dataset is applied to this model, i.e., the test dataset, the classification accuracy of the model demonstrated that the model provides desirable qualities with the classification accuracy of $97 \%$. It worth noting that, when an independent testing data is applied, the DT classifier provides a high classification accuracy of $98 \%$. The $k$-NN provide the second highest training classification accuracy with $87 \%$ and a testing accuracy of $81 \%$ recorded and $81 \%$ for the independent testing (validation). Meanwhile, for other classifier models i.e., SVM, LDA, and LR provide the same accuracy for both training and testing demonstrated that the classifiers have reached its saturation stage with the default hyperparameters of the models. It worth noting that, even though the training time and prediction time of LDA model is fastest among others classifier, the training accuracy for this model is low compare to the DT model. Fig. 7 depicts the classification accuracies across all models developed. 
259

260

261

262

263

264

265

266

267

268

269

270

271

272

273

274

275

276

277

278

279

280

281

282

283

284

285

286

287

288

289

290

291

292

293

294

295

296

297

298

A similar observation could be seen in Table 2, in which only the significant features identified by ERT, i.e., MIN and MAX are used. It is apparent that a comparable performance is illustrated, however, it is worth noting that the training time, as well as the prediction speed of the classifier, has been improved. It is apparent from Fig. 8 that the DT and k-NN model demonstrated desirable qualities with better classification accuracies for training, testing and validation. The reduction of the features is non-trivial especially for real-time implementation as the computational expense could be significantly reduced.

The present findings have demonstrated that through selected significant features, a comparable classification accuracy is attainable. It should be noted that a better $\mathrm{CA}$ is reported in the present study in comparison to a similar investigation carried out by Gandolla et al. (2017). The authors employed Artificial Neural Network in classifying different motions extracted from EMG signals. The study reported an average testing CA of $76 \%$ was obtained in correctly predicting healthy subjects' motion intention. In addition, an EMG-based motion intention classification of different reaching movement was investigated by Cesqui et al. (2013). It was shown from the study that the SVM architecture utilised of significant features identified could only yield a classification accuracy of $97.5 \%$. Therefore, it could be clearly shown that the proposed technique employed in the present investigation could yield a reasonably well CA of motion intention.

\section{Conclusions}

This paper presented an approach to detect and capture the subject's intention through the EMG signal. From the EMG signal processed a number of features were extracted for the classification purpose, namely WL, MAV, RMS, SD, MIN and MAX. Then a feature selection method was introduced in this study in order to get the significant features by means of Extremely Randomised Tree technique. The features extracted upon investigating the feature importance, are MAX and MIN, respectively. It was demonstrated from the present investigation that the DT classifier yielded an excellent classification with a classification accuracy of $100 \%, 99 \%$ and 99 $\%$ on training, testing and validation dataset. Future works will focus on developing a controller for a rehabilitation robot based on the output of the classifier from the EMG signal taken from the subject

\section{Acknowledgements}

The work presented was carried out in the Biomechatronics Research Laboratory of International Islamic University Malaysia. The authors wish to gratefully acknowledge the FRGS grant funding (FRGS17-029-0595) from the Ministry of Higher Education Malaysia.

\section{References}

AlOmari F, Liu G. 2014. Analysis of extracted forearm sEMG signal using LDA, QDA, K-NN classification algorithms. Open Automation and Control Systems Journal 6:108-116. DOI: 
10.2174/1874444301406010108.

Bhattacharya A, Sarkar A, Basak P. 2017. Time domain multi-feature extraction and classification of human hand movements using surface EMG. In: 2017 4th International Conference on Advanced Computing and Communication Systems, ICACCS 2017. 1-5. DOI: 10.1109/ICACCS.2017.8014594.

Bi L, Feleke A, Guan C. 2019. A review on EMG-based motor intention prediction of continuous human upper limb motion for human-robot collaboration. Biomedical Signal Processing and Control 51:113-127. DOI: 10.1016/j.bspc.2019.02.011.

Brackenridge J, Bradnam L V., Lennon S, Costi J. J., Hobbs DA. 2016. A Review of Rehabilitation Devices to Promote Upper Limb Function Following Stroke. Neuroscience and Biomedical Engineering 4:25-42. DOI: 10.2174/2213385204666160303220102.

Cesqui B, Tropea P, Micera S, Krebs HI. 2013. EMG-based pattern recognition approach in post stroke robot-aided rehabilitation: A feasibility study. Journal of NeuroEngineering and Rehabilitation 10:1-15. DOI: 10.1186/1743-0003-10-75.

Chen S-H, Lien W-M, Wang W-W, Lee G-D, Hsu L-C, Lee K-W, Lin S-Y, Lin C-H, Fu L-C, Lai J-S, Luh J-J, Chen W-S. 2016. Assistive Control System for Upper Limb Rehabilitation Robot. IEEE transactions on neural systems and rehabilitation engineering : a publication of the IEEE Engineering in Medicine and Biology Society 24:1199-1209. DOI: 10.1109/TNSRE.2016.2532478.

Colombo R, Pisano F, Micera S, Mazzone A, Delconte C, Chiara Carrozza M, Dario P, Minuco G. 2005. Robotic techniques for upper limb evaluation and rehabilitation of stroke patients. IEEE Transactions on Neural Systems and Rehabilitation Engineering 13:311-324. DOI: 10.1109/TNSRE.2005.848352.

Fasoli SE. 2016. Rehabilitation Technologies to Promote Upper Limb Recovery after Stroke. Elsevier Ltd. DOI: 10.1016/B978-0-323-17281-3.00022-8.

Gandolla M, Ferrante S, Ferrigno G, Baldassini D, Molteni F, Guanziroli E, Cotti Cottini M, Seneci C, Pedrocchi A. 2017. Artificial neural network EMG classifier for functional hand grasp movements prediction. Journal of International Medical Research 45:1831-1847. DOI: $10.1177 / 0300060516656689$.

Geurts P, Ernst D, Wehenkel L. 2006. Extremely randomized trees. Machine learning 63:3-42.

Guidali M, Keller U, Klamroth-Marganska V, Nef T, Riener R. 2013. Estimating the patient's contribution during robot-assisted therapy. Journal of Rehabilitation Research and Development 50:379-394. DOI: 10.1682/JRRD.2011.09.0172.

Huang J, Tu X, He J. 2016. Design and Evaluation of the RUPERT Wearable Upper Extremity Exoskeleton Robot for Clinical and In-Home Therapies. IEEE Transactions on Systems, Man, and Cybernetics: Systems 46:926-935. DOI: 10.1109/TSMC.2015.2497205.

Kamavuako EN, Scheme EJ, Englehart KB. 2014. Combined surface and intramuscular EMG for improved real-time myoelectric control performance. Biomedical Signal Processing and Control 10:102-107. DOI: 10.1016/j.bspc.2014.01.007.

Kosaki T, Atsuumi K, Takahashi Y, Li S. 2017. A pneumatic arm power-assist system prototype with EMG-based muscle activity detection. 2017 IEEE International Conference on Mechatronics and Automation, ICMA 2017:793-798. DOI: 10.1109/ICMA.2017.8015917.

Krebs HI, Palazzolo JJ, Dipietro L, Ferraro M, Krol J, Rannekleiv K, Volpe BT, Hogan N. 2003. Rehabilitation robotics: Performance-based progressive robot-assisted therapy. Autonomous Robots 15:7-20. DOI: 10.1023/A:1024494031121.

Loureiro RCV, Lamperd B, Collin C, Harwin WS. 2009. Reach \& grasp therapy: Effects of the 
345

346

347

348

349

350

351

352

353

354

355

356

357

358

359

360

361

362

363

364

365

366

367

368

369

370

371

372

373

374

375

376

377

378

379

380

381

382

383

384

385

386

387

388

389

390
gentle/G system assessing sub-acute stroke whole-arm rehabilitation. 2009 IEEE International Conference on Rehabilitation Robotics, ICORR 2009:755-760. DOI: 10.1109/ICORR.2009.5209509.

Marchal-Crespo L, Reinkensmeyer DJ. 2009. Review of control strategies for robotic movement training after neurologic injury. Journal of neuroengineering and rehabilitation 6:20. DOI: 10.1186/1743-0003-6-20.

Naik G. 2015. Applications, Challenges, and Advancements in Electromyography Signal Processing. DOI: 10.4018/978-1-4666-6090-8.

Oskoei MA, Hu H. 2008. Support vector machine-based classification scheme for myoelectric control applied to upper limb. IEEE Transactions on Biomedical Engineering 55:19561965. DOI: 10.1109/TBME.2008.919734.

Peternel L, Noda T, Petrič T, Ude A, Morimoto J, Babi?? J. 2016. Adaptive control of exoskeleton robots for periodic assistive behaviours based on EMG feedback minimisation. PLoS ONE 11:1-26. DOI: 10.1371/journal.pone.0148942.

Proietti T, Crocher V, Roby-Brami A, Jarrasse N. 2016. Upper-Limb Robotic Exoskeletons for Neurorehabilitation: A Review on Control Strategies. IEEE Reviews in Biomedical Engineering 9:4-14. DOI: 10.1109/RBME.2016.2552201.

Purushothaman G, Ray KK. 2014. EMG based man-machine interaction - A pattern recognition research platform. Robotics and Autonomous Systems 62:864-870. DOI: 10.1016/j.robot.2014.01.008.

Rahman MH, Ochoa-Luna C, Saad M. 2015a. EMG Based Control of a Robotic Exoskeleton for Shoulder and Elbow Motion Assist. Journal of Automation and Control Engineering 3:270276. DOI: $10.12720 /$ joace.3.4.270-276.

Rahman MH, Ochoa-Luna C, Saad M. 2015b. EMG Based Control of a Robotic Exoskeleton for Shoulder and Elbow Motion Assist. Journal of Automation and Control Engineering 3:270276. DOI: 10.12720/joace.3.4.270-276.

Rahman MH, Ochoa-luna C, Saad M, Archambault P. 2015. Motion Control of an Exoskeleton Robot using Electromyogram Signals. Advances in Robotics, Mechatronics and Circuits:27-33.

Razman MAM, Susto GA, Cenedese A, Majeed APPA, Musa RM, Ghani ASA, Adnan FA, Ismail KM, Taha Z, Mukai Y. 2019. Hunger classification of Lates calcarifer by means of an automated feeder and image processing. Computers and Electronics in Agriculture 163:104883.

Rechy-Ramirez EJ, Hu H. 2015. Bio-signal based control in assistive robots: a survey. Digital Communications and Networks 1:85-101. DOI: 10.1016/j.dcan.2015.02.004.

Spiewak C. 2018. A Comprehensive Study on EMG Feature Extraction and Classifiers. Open Access Journal of Biomedical Engineering and Biosciences 1:1-10. DOI: 10.32474/oajbeb.2018.01.000104.

Taha Z, Abdul Majeed APP, Abdullah MA, Azmi KZM, Zakaria MA Bin, Ghani ASA, Hassan MHA, Razman MAM. 2019. The Control of an Upper Extremity Exoskeleton for Stroke Rehabilitation by Means of a Hybrid Active Force Control. In: 361-370. DOI: 10.1007/9783-319-78452-6_30.

Tommaso Lenzi, Stefano Marco Maria De Rossi, Nicola Vitiello Maria CC. 2012. Intentionbased EMG Control for Powered Exoskeletons. 59:2180-2190.

Veer K, Sharma T. 2016. A novel feature extraction for robust EMG pattern recognition. Journal of Medical Engineering and Technology 40:149-154. DOI:

Peer] Comput. Sci. reviewing PDF | (CS-2020:10:54392:1:1:NEW 31 Dec 2020) 
391

392

393

394

395

396

397

398
10.3109/03091902.2016.1153739.

Winter D a. 1990. Biomechanics and Motor Control of Human Movement. DOI: 10.1002/9780470549148.

Zimmermann R, Marchal-Crespo L, Lambercy O, Fluet M-C, Metzger J-C, Edelmann J, Brand J, Eng K, Riener R, Wolf M, Gassert R. 2015. What's Your Next Move? Detecting Movement Intention for Stroke Rehabilitation. Biosystems \& biorobotics, 109 pages. DOI: 10.1007/978-3-319-25190-5. 
Figure 1

Block Diagram for data acquisition

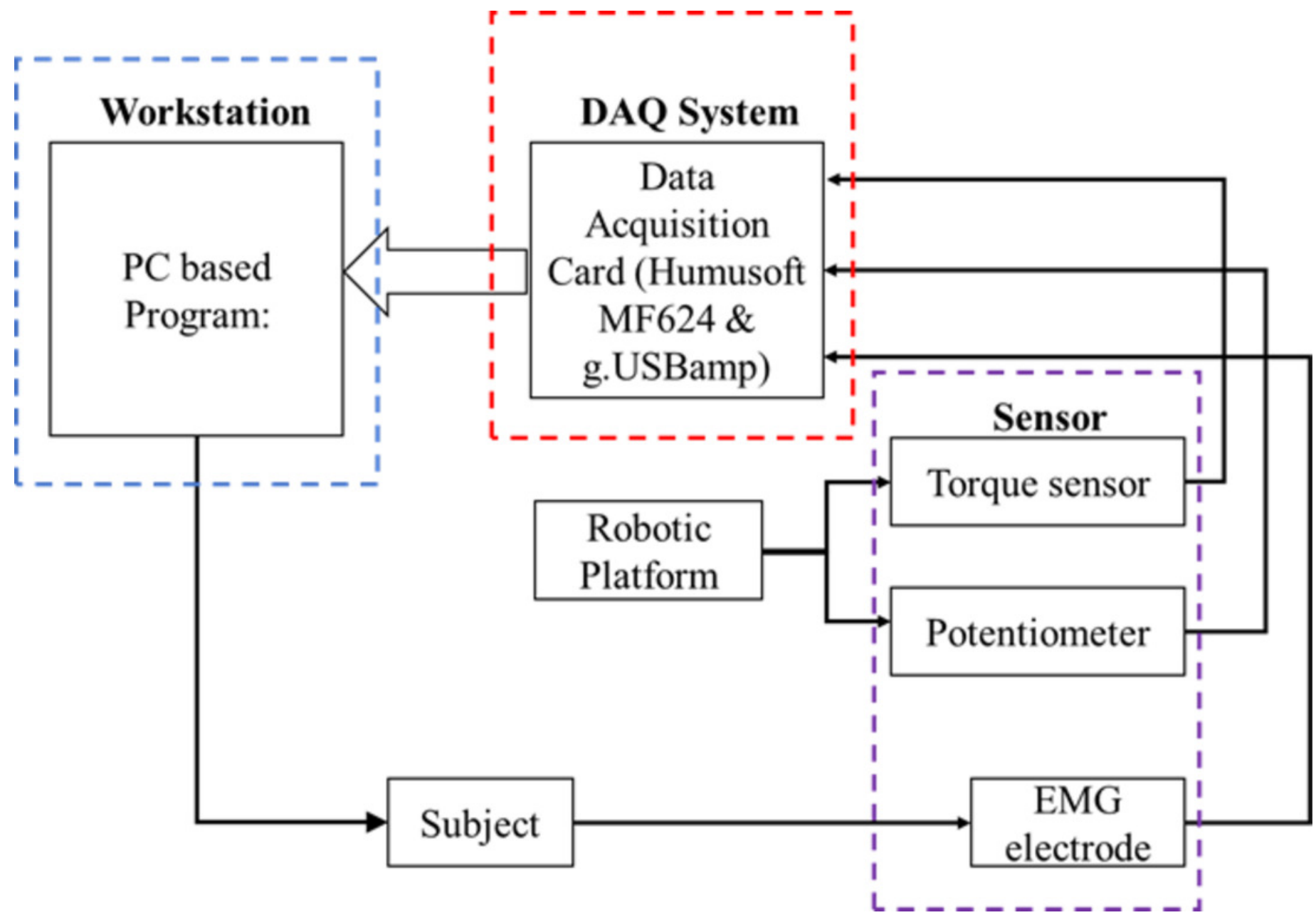


Figure 2

Robotic Platform 


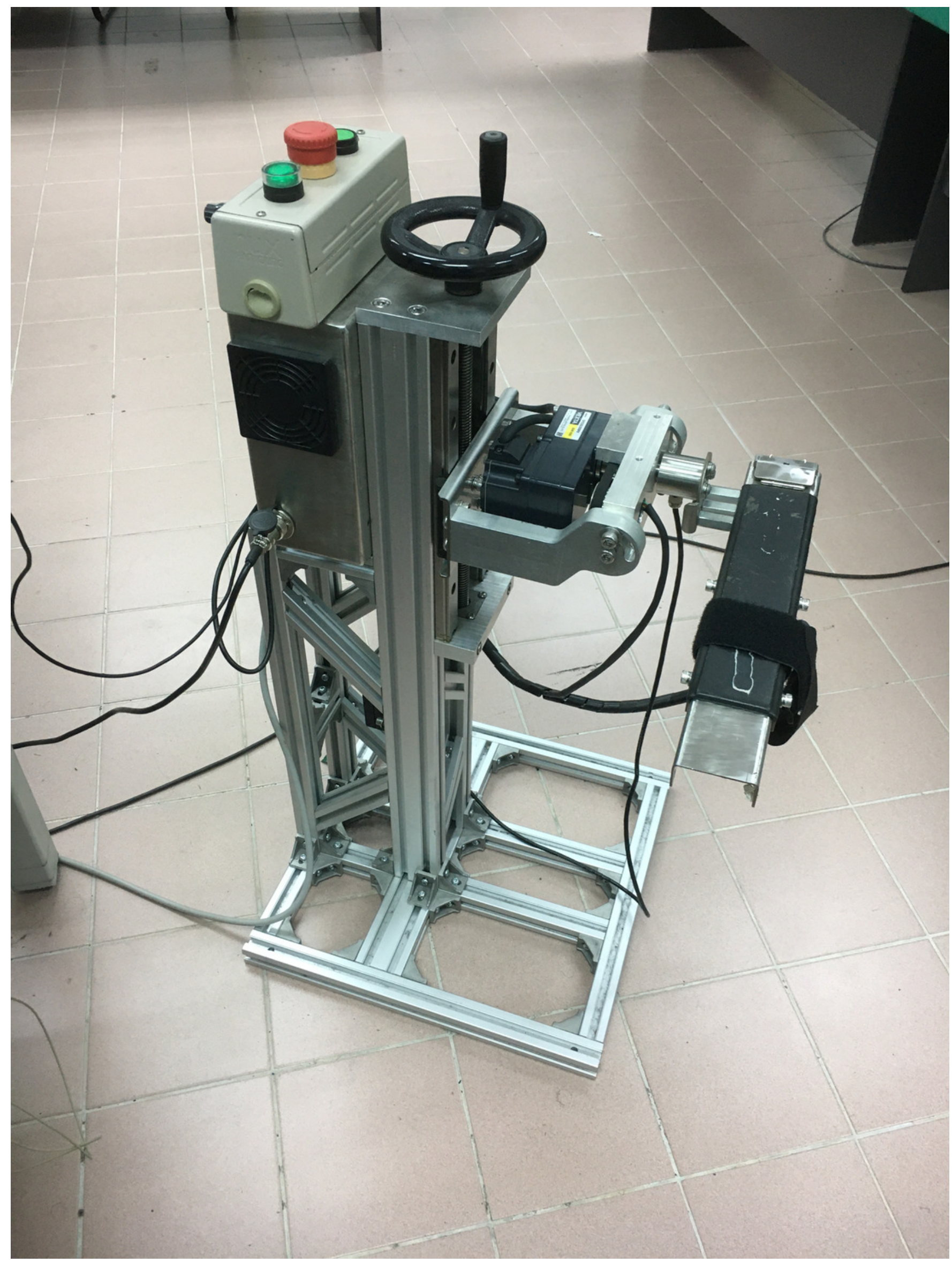




\section{Figure 3}

\section{Electrode placement}

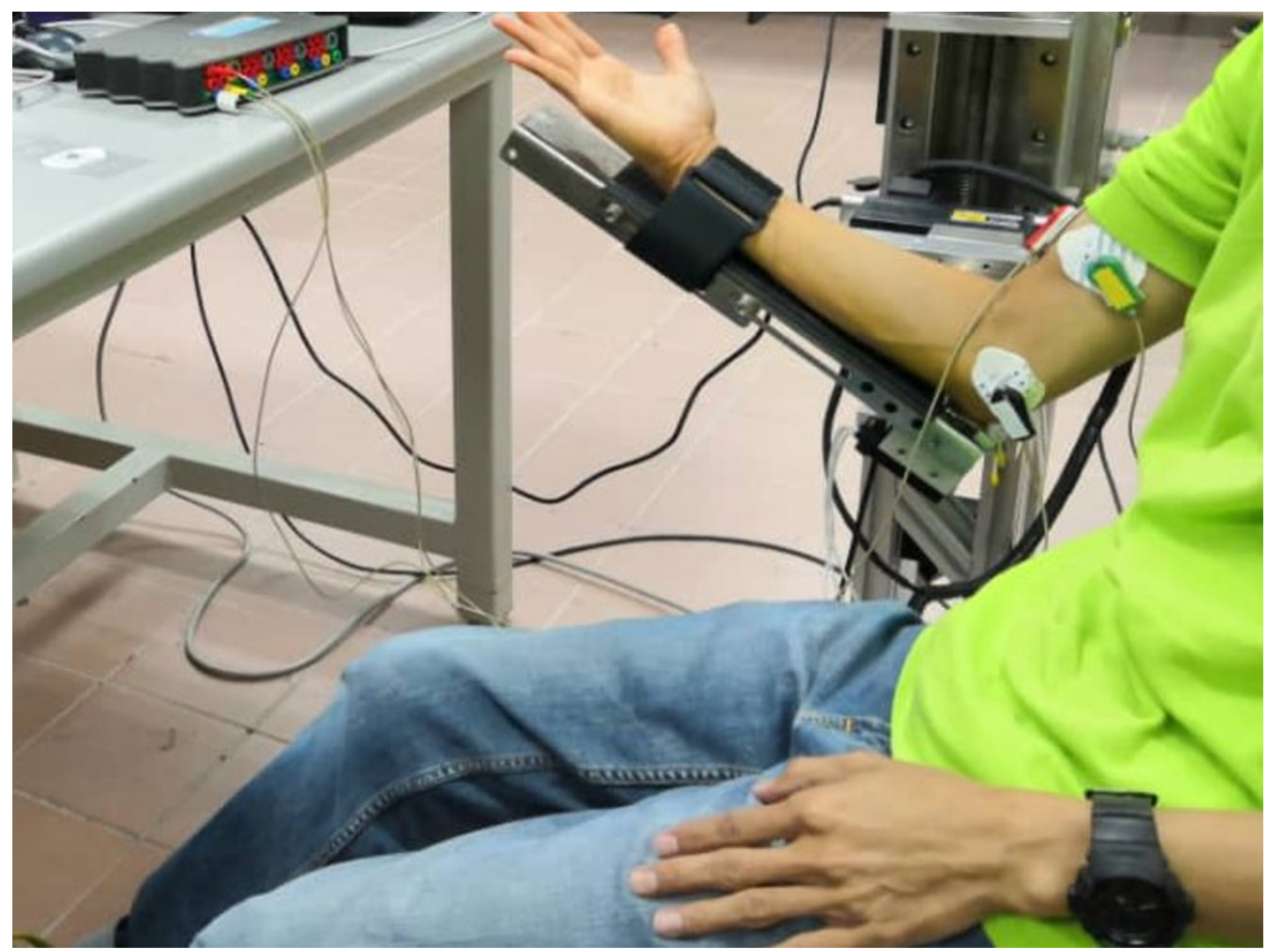


Figure 4

A sample of the EMG and angular position for a single movement

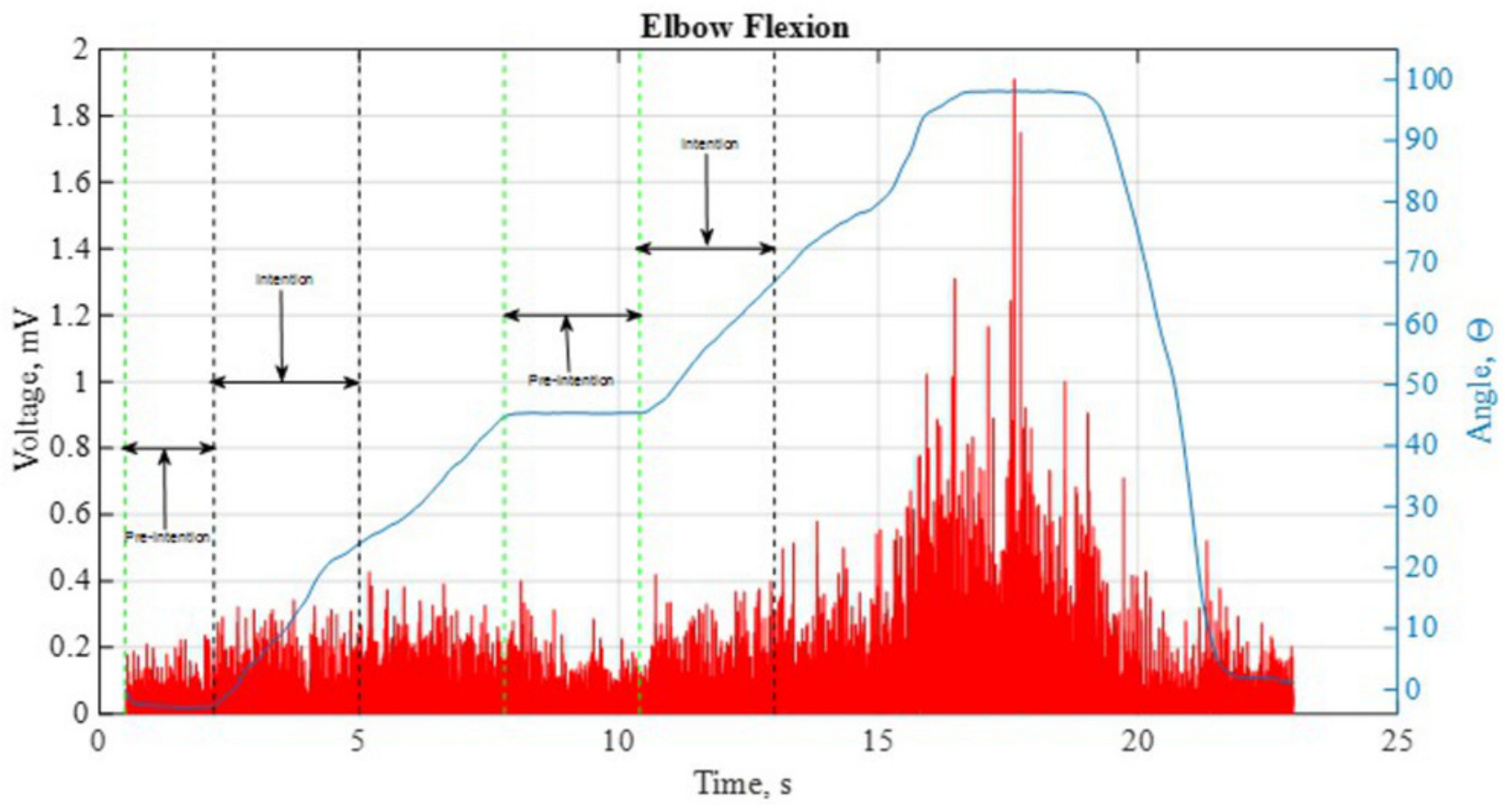


Figure 5

Bar graph of feature importance

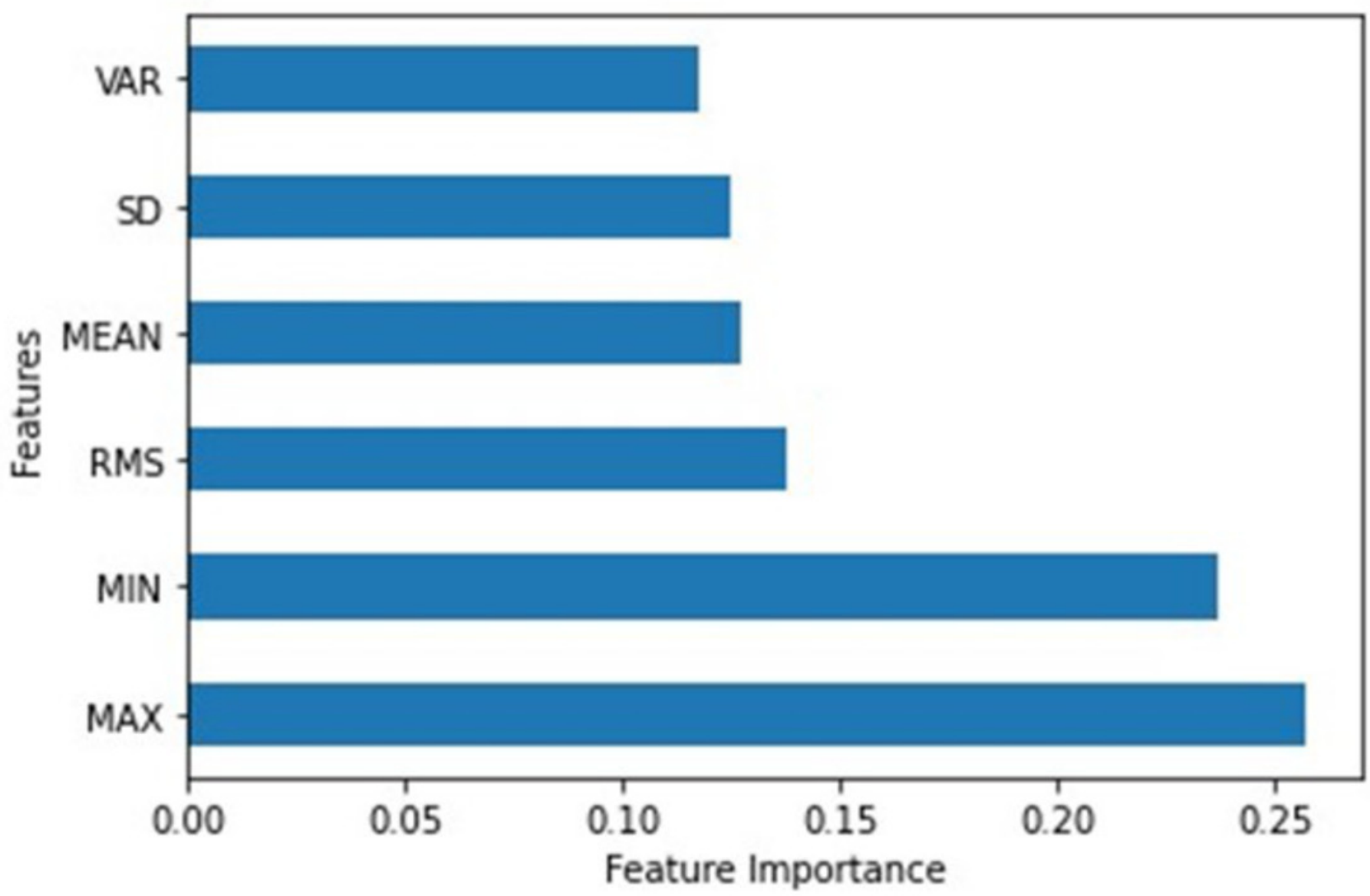


Figure 6

Confusion matrix

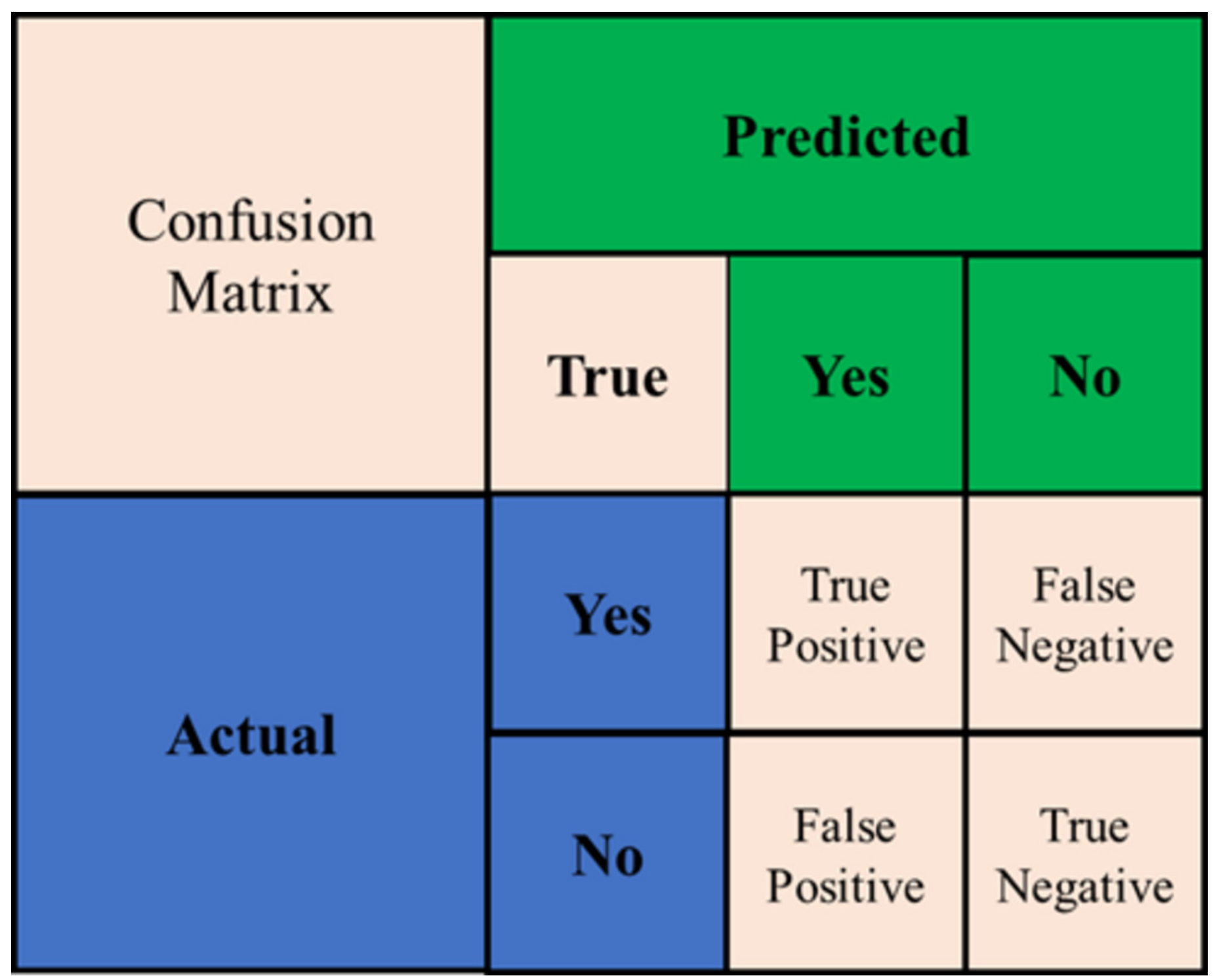




\section{Figure 7}

Classification accuracy for each model for all features

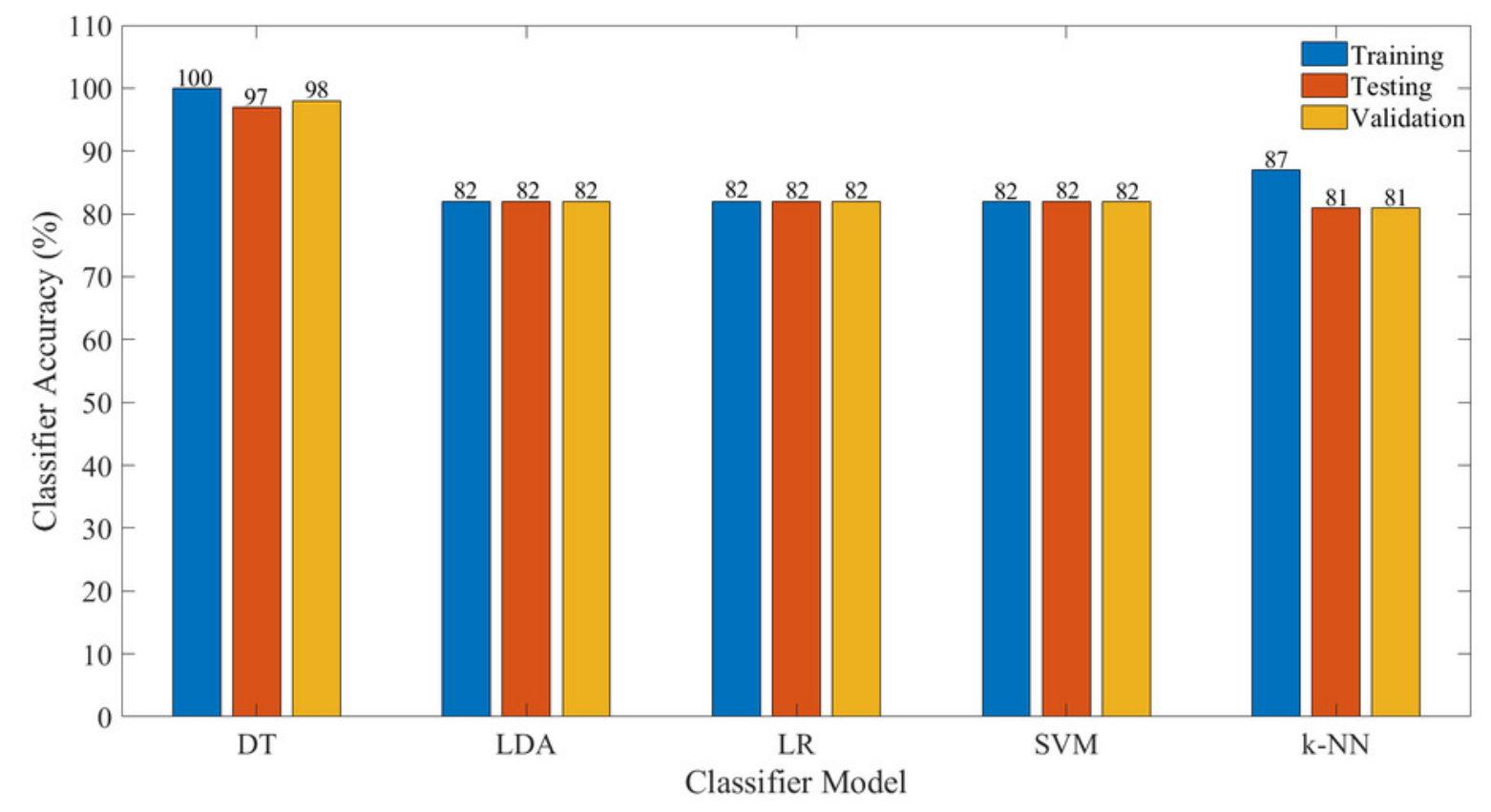


Figure 8

Classification accuracy for each model for the selected features

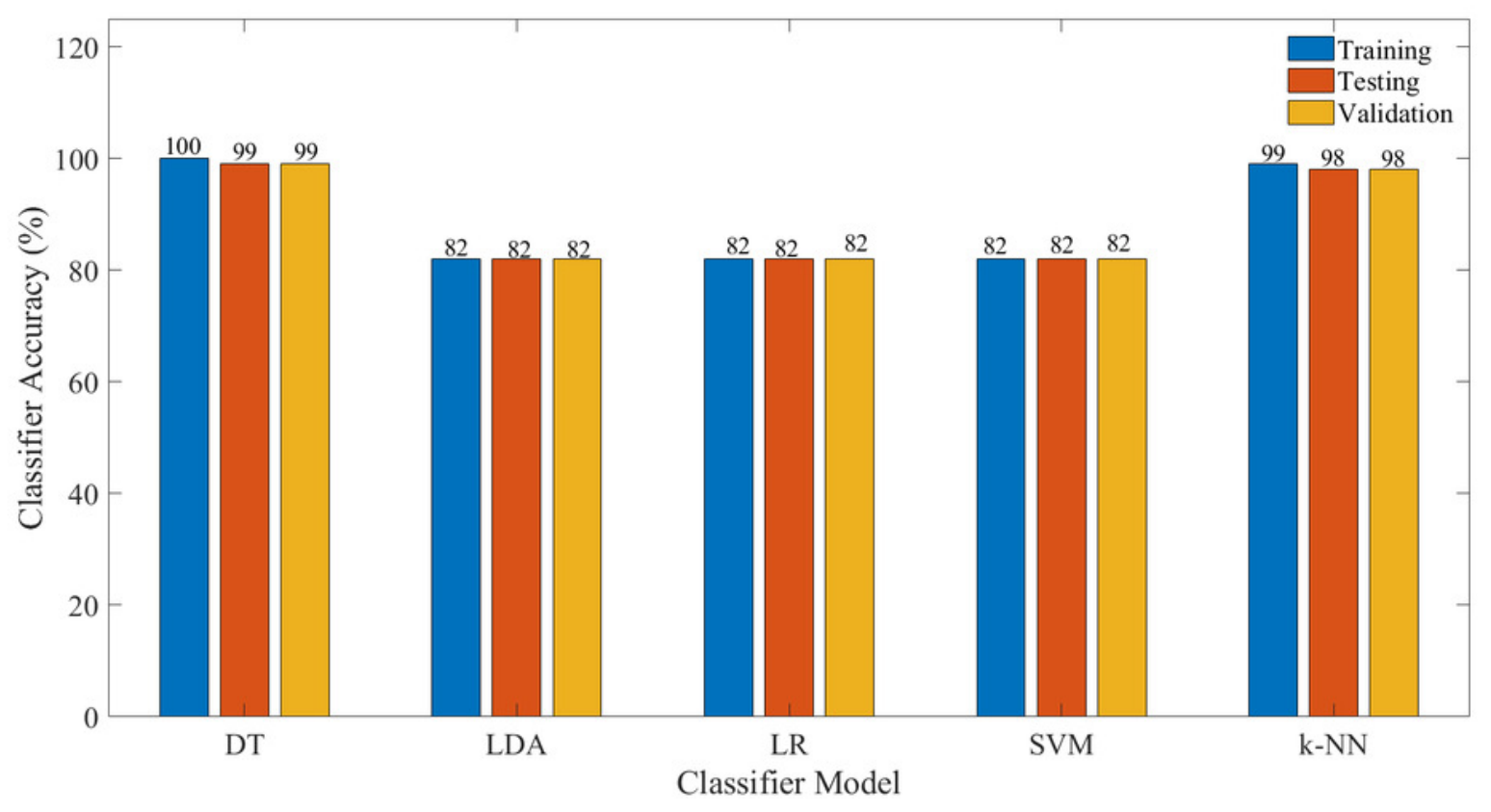




\section{Table $\mathbf{1}$ (on next page)}

Classifier models performance with all features 
1

\begin{tabular}{|c|c|c|c|c|c|c|c|c|c|c|c|c|}
\hline \multirow{2}{*}{$\begin{array}{c}\text { Classifier } \\
\text { model }\end{array}$} & \multirow{2}{*}{$\begin{array}{c}\text { Training } \\
\text { time (s) }\end{array}$} & \multirow{2}{*}{$\begin{array}{l}\text { Prediction } \\
\text { time (s) }\end{array}$} & \multicolumn{4}{|c|}{ Train (\%) } & \multicolumn{6}{|c|}{ Test (\%) } \\
\hline & & & Prec & Recall & F1 & Acc & Prec & Recall & $\mathbf{F}_{1}$ & Acc & $\begin{array}{r}\text { Conf } \\
\text { Ma }\end{array}$ & $\begin{array}{l}\text { sion } \\
\text { rix }\end{array}$ \\
\hline \multirow{2}{*}{$k-\mathrm{NN}$} & \multirow{2}{*}{0.947} & \multirow{2}{*}{9.121} & \multirow{2}{*}{88} & \multirow{2}{*}{96} & \multirow{2}{*}{92} & \multirow{2}{*}{87} & \multirow{2}{*}{85} & \multirow{2}{*}{93} & \multirow{2}{*}{89} & \multirow{2}{*}{81} & 34460 & 2590 \\
\hline & & & & & & & & & & & 6026 & 2282 \\
\hline \multirow{2}{*}{ SVM } & \multirow{2}{*}{861.168} & \multirow{2}{*}{290.256} & \multirow{2}{*}{82} & \multirow{2}{*}{100} & \multirow{2}{*}{90} & \multirow{2}{*}{82} & \multirow{2}{*}{82} & \multirow{2}{*}{100} & \multirow{2}{*}{90} & \multirow{2}{*}{82} & 37049 & 1 \\
\hline & & & & & & & & & & & 8308 & 0 \\
\hline \multirow{2}{*}{ LDA } & \multirow{2}{*}{0.221} & \multirow{2}{*}{0.006} & \multirow{2}{*}{82} & \multirow{2}{*}{100} & \multirow{2}{*}{90} & \multirow{2}{*}{82} & \multirow{2}{*}{82} & \multirow{2}{*}{100} & \multirow{2}{*}{90} & & 37049 & 1 \\
\hline & & & & & & & & & & 82 & 8308 & 0 \\
\hline DT & 3087 & 0051 & 100 & 100 & 100 & 100 & 08 & 08 & 08 & 07 & 36470 & 580 \\
\hline D1 & 3.081 & 0.051 & 100 & 100 & 100 & 100 & 98 & 98 & 98 & 91 & 569 & 7739 \\
\hline L R & 2115 & 0007 & 82 & 100 & 90 & 82 & 82 & 100 & 90 & 82 & 37050 & 0 \\
\hline & & & & & & & & 100 & 90 & 82 & 8308 & 0 \\
\hline
\end{tabular}

2

3

\begin{tabular}{|c|c|c|c|c|c|c|}
\hline \multirow{2}{*}{$\begin{array}{c}\text { Classifier } \\
\text { model }\end{array}$} & \multicolumn{6}{|c|}{ Validation (\%) } \\
\hline & Prec & Recall & $\mathbf{F}_{1}$ & Acc & \multicolumn{2}{|c|}{$\begin{array}{c}\text { Confusion } \\
\text { Matrix }\end{array}$} \\
\hline \multirow{2}{*}{$k-\mathrm{NN}$} & \multirow{2}{*}{85} & \multirow{2}{*}{93} & \multirow{2}{*}{89} & \multirow{2}{*}{81} & 34460 & 2589 \\
\hline & & & & & 6031 & 2277 \\
\hline \multirow{2}{*}{ SVM } & \multirow{2}{*}{82} & \multirow{2}{*}{100} & \multirow{2}{*}{90} & \multirow{2}{*}{82} & 37049 & 0 \\
\hline & & & & & 8308 & 0 \\
\hline \multirow{2}{*}{ LDA } & \multirow{2}{*}{82} & \multirow{2}{*}{100} & \multirow{2}{*}{90} & \multirow{2}{*}{82} & 37049 & 0 \\
\hline & & & & & 8308 & 0 \\
\hline \multirow{2}{*}{ DT } & \multirow{2}{*}{98} & \multirow{2}{*}{99} & \multirow{2}{*}{99} & \multirow{2}{*}{98} & 36519 & 530 \\
\hline & & & & & 576 & 7732 \\
\hline \multirow{2}{*}{ LR } & \multirow{2}{*}{82} & \multirow{2}{*}{100} & \multirow{2}{*}{90} & \multirow{2}{*}{82} & 37049 & 0 \\
\hline & & & & & 8308 & 0 \\
\hline
\end{tabular}

4

5

Table 1 Classifier models performance with all features 


\section{Table 2 (on next page)}

Classifier models performance with selected features 
1

\begin{tabular}{|c|c|c|c|c|c|c|c|c|c|c|c|c|}
\hline \multirow{2}{*}{$\begin{array}{c}\text { Classifier } \\
\text { model }\end{array}$} & \multirow{2}{*}{$\begin{array}{c}\text { Training } \\
\text { time (s) }\end{array}$} & \multirow{2}{*}{$\begin{array}{l}\text { Prediction } \\
\text { time (s) }\end{array}$} & \multicolumn{4}{|c|}{ Train (\%) } & \multicolumn{6}{|c|}{ Test (\%) } \\
\hline & & & Prec & Recall & F1 & Acc & Prec & Recall & $\mathbf{F}_{1}$ & Acc & $\begin{array}{r}\text { Conf } \\
\text { Ma }\end{array}$ & $\begin{array}{l}\text { sion } \\
\text { ix }\end{array}$ \\
\hline \multirow{2}{*}{$k-\mathrm{NN}$} & \multirow{2}{*}{0.289} & \multirow{2}{*}{5.798} & \multirow{2}{*}{99} & \multirow{2}{*}{99} & \multirow{2}{*}{99} & \multirow{2}{*}{99} & \multirow{2}{*}{99} & \multirow{2}{*}{99} & \multirow{2}{*}{99} & \multirow{2}{*}{98} & 36634 & 416 \\
\hline & & & & & & & & & & & 434 & 7874 \\
\hline \multirow{2}{*}{ SVM } & \multirow{2}{*}{385.365} & \multirow{2}{*}{233.06} & \multirow{2}{*}{82} & \multirow{2}{*}{100} & \multirow{2}{*}{90} & \multirow{2}{*}{82} & \multirow{2}{*}{82} & \multirow{2}{*}{100} & \multirow{2}{*}{90} & \multirow{2}{*}{82} & 37050 & 0 \\
\hline & & & & & & & & & & & 8308 & 0 \\
\hline \multirow{2}{*}{ LDA } & \multirow{2}{*}{0.09} & \multirow{2}{*}{0.008} & \multirow{2}{*}{82} & \multirow{2}{*}{100} & \multirow{2}{*}{90} & \multirow{2}{*}{82} & \multirow{2}{*}{82} & \multirow{2}{*}{100} & \multirow{2}{*}{90} & & 37050 & 0 \\
\hline & & & & & & & & & & 82 & 8308 & 0 \\
\hline DT & 0585 & 0053 & 100 & 100 & 100 & 100 & 09 & 09 & 09 & 09 & 36864 & 186 \\
\hline D1 & 0.585 & 0.053 & 100 & 100 & 100 & 100 & 99 & 99 & 99 & 99 & 199 & 8109 \\
\hline LR & 0686 & 0004 & 82 & 100 & 90 & 82 & 82 & 100 & 90 & 82 & 37050 & 0 \\
\hline & & & & & & & & 100 & 90 & 82 & 8308 & 0 \\
\hline
\end{tabular}

2

\begin{tabular}{|c|c|c|c|c|c|c|}
\hline \multirow{2}{*}{$\begin{array}{c}\text { Classifier } \\
\text { model }\end{array}$} & \multicolumn{6}{|c|}{ Validation (\%) } \\
\hline & Prec & Recall & $F_{1}$ & Acc & \multicolumn{2}{|c|}{$\begin{array}{c}\text { Confusion } \\
\text { Matrix }\end{array}$} \\
\hline \multirow{2}{*}{$k-\mathrm{NN}$} & \multirow{2}{*}{99} & \multirow{2}{*}{99} & \multirow{2}{*}{99} & \multirow{2}{*}{98} & 36676 & 373 \\
\hline & & & & & 414 & 7894 \\
\hline \multirow{2}{*}{ SVM } & \multirow{2}{*}{82} & \multirow{2}{*}{100} & \multirow{2}{*}{90} & \multirow{2}{*}{82} & 37049 & 0 \\
\hline & & & & & 8308 & 0 \\
\hline \multirow{2}{*}{ LDA } & \multirow{2}{*}{82} & \multirow{2}{*}{100} & \multirow{2}{*}{90} & \multirow{2}{*}{82} & 37049 & 0 \\
\hline & & & & & 8308 & 0 \\
\hline \multirow{2}{*}{ DT } & \multirow{2}{*}{99} & \multirow{2}{*}{99} & \multirow{2}{*}{99} & \multirow{2}{*}{99} & 36863 & 186 \\
\hline & & & & & 188 & 8120 \\
\hline \multirow{2}{*}{ LR } & \multirow{2}{*}{82} & \multirow{2}{*}{100} & \multirow{2}{*}{90} & \multirow{2}{*}{82} & 37049 & 0 \\
\hline & & & & & 8308 & 0 \\
\hline
\end{tabular}

Table 2 Classifier models performance with the selected features 